\title{
ON THE FREQUENCY OF SMALL FRACTIONAL PARTS IN CERTAIN REAL SEQUENCES. II
}

BY

W. J. LEVEQUE

1. Introduction. Let $\langle x\rangle$ be the distance between $x$ and the integer nearest $x$, let $f$ be a decreasing function on the positive real numbers, with

(1) $f(x)=O\left(x^{-1}\right), f^{\prime}(x)=O\left(x^{-2}\right)$ as $x \rightarrow \infty ; 0<f(x)<1 / 2 ; \sum_{k=1}^{\infty} f(k)=\infty$;

and let $g(x)=f(\log x) / x$ and $G(n)=\sum_{1}^{n} g(k)$. In a recent paper [2] in this journal I claimed to have proved the following assertions:

I. Let

$$
T_{n}(x)=\text { No }\{m \leqq n \mid\langle m x\rangle<g(m)\} ;
$$

then $T_{n}$ has a limiting normal distribution, in the sense that for fixed $\omega$,

$$
\begin{aligned}
\lim _{n \rightarrow \infty} \text { meas }\left\{x \in[0,1] \mid T_{n}(x)<12 \pi^{-2} G(n)\right. & \left.+\omega\left(12 \pi^{-2} G(n)\right)^{1 / 2}\right\} \\
& =\phi(\omega)=(2 \pi)^{-1 / 2} \int_{-\infty}^{\omega} e^{-u^{2} / 2} d u .
\end{aligned}
$$

II. For almost all $x$,

$$
T_{n}(x) \sim 12 \pi^{-2} G(n) .
$$

Professor Erdös has pointed out to me that I have not in fact proved I and II, but rather the following, where $l_{m}=l_{m}(x)$ is the integer nearest $m x$.

I'. Let

$$
U_{n}=\text { No }\left\{m \leqq n \mid\langle m x\rangle\left\langle g(m),\left(m, l_{m}\right)=1\right\} .\right.
$$

Then (2) holds with $T_{n}$ replaced by $U_{n}$.

II'. For almost all $x$,

$$
U_{n}(x) \sim 12 \pi^{-2} G(n) .
$$

The error arose on p. 246 of [2], when I used the well-known theorem that if $|m x-l|<1 / 2 m$, then $l / m$ is a convergent $p_{k} / q_{k}$ to $x$, to deduce incorrectly that

(4) No $\left\{m \leqq q_{n} \mid\langle m x\rangle<\frac{f(\log m)}{m}\right\}=$ No $\left\{k \leqq n|| q_{k} x-p_{k} \mid<\frac{f\left(\log q_{k}\right)}{q_{k}}\right\}$,

Received by the editors November 1, 1958. 
whereas in fact it follows only that

$$
\text { No } \begin{aligned}
\left\{m \leqq q_{n} \mid\langle m x\rangle\right. & \left.<\frac{f(\log m)}{m}\right\} \\
& =\text { No }\left\{k, r|| r q_{k} x-r p_{k} \mid<\frac{f\left(\log r q_{k}\right)}{r q_{k}}, 1 \leqq r q_{k} \leqq q_{n}\right\} .
\end{aligned}
$$

The right member of (4) is exactly $U_{n}$, and the proof in [2] leads directly to $\mathrm{I}^{\prime}$ and $\mathrm{II}^{\prime}$.

There was an anomaly in relations (2) and (3) in the presence of the factor $6 / \pi^{2}$, since on probabilistic grounds one would expect the coefficient of $G(n)$ to be 2 . This can now be explained heuristically by the known fact that

$$
\text { No }\{k, l \mid k \leqq n, l \leqq n,(k, l)=1\} \sim 6 \pi^{-2} \text { No }\{k, l \mid k \leqq n, l \leqq n\},
$$

or that, roughly speaking, the probability that two positive integers chosen at random be relatively prime is $6 / \pi^{2}$. Such considerations suggest that the following assertions may hold:

$I^{\prime \prime} . \lim _{n \rightarrow \infty}$ meas $\left\{x \in[0,1] \mid T_{n}(x)<2 G(n)+\omega(2 G(n))^{1 / 2}\right\}=\phi(\omega)$.

II". For almost all $x$,

$$
T_{n}(x) \sim 2 G(n) .
$$

I have not been able to prove $I^{\prime \prime}$, but in $\$ 2$ I obtain an approximation to it. In the course of reconsidering these questions $I$ have noticed that a false implication was used in the proof of $I^{\prime}$, the conclusion of which is true in the case at hand but requires a different proof. This is also rectified in $\$ 2$.

In $\$ \S 3-4, \mathrm{II}^{\prime \prime}$ is proved, and in fact under much weaker hypotheses on the function $g$. Since the proof of $\mathrm{II}^{\prime}$ was not given in detail, it being a trivial modification of that of $\mathrm{I}^{\prime}$, and since the proof of the new theorem differs in several respects, it is given in full.

We use the notation of [2] when convenient.

2. Corrections. Part (f) of Lemma 1 of [2] is false. However, the following substitute suffices:

Let $f$ be a decreasing function on the positive real numbers, satisfying (1). If $\alpha_{1}, \alpha_{2}, \cdots$ and $\alpha$ are arbitrary numbers such that

$$
\sum_{k=1}^{n} \alpha_{k}=n \alpha+O\left(n^{\delta}\right)
$$

as $n \rightarrow \infty$, then

$$
\sum_{k=1}^{n} \alpha_{k} f(k)=\alpha \sum_{k=1}^{n} f(k)+O(1)
$$


For brevity let $\alpha_{k}-\alpha=b_{k}$, put $B_{k}=\sum_{1}^{k} b_{j}$, and write

$$
\sum_{k=1}^{n} b_{k} f(k)=\sum_{k=1}^{n-1} B_{k}(f(k)-f(k+1))+B_{n} f(n) .
$$

The result follows on noting that

$$
B_{n} f(n)=O\left(n^{\delta}\right) O\left(n^{-1}\right)=o(1)
$$

and

$$
\begin{aligned}
\left|\sum_{k=1}^{n-1} B_{k}(f(k)-f(k+1))\right| & \leqq c \sum_{k=1}^{n-1} k^{\delta}(f(k)-f(k+1)) \\
& =c \sum_{k=1}^{n-1} k^{\delta} O\left(k^{-2}\right)=O(1),
\end{aligned}
$$

since

$$
f(k)-f(k+1)=-\int_{k}^{k+1} f^{\prime}(t) d t=O\left(k^{-2}\right) .
$$

Lemma $1(f)$ of [2] was used only to prove that for almost all $x$,

$$
\sum_{k=1}^{n} f(k)\left(1+\frac{q_{k-1}}{q_{k}}\right)=\frac{1}{\log 2} \sum_{k=1}^{n} f(k)+O(1),
$$

where $q_{k}$ is the denominator of the $k$ th convergent to the continued fraction expansion of $x$. To obtain (5) from the substitute lemma, we need the following result. that

Lemma 1. If $\delta<1 / 2$, then for almost every $x$ there is a number $\lambda=\lambda(x)$ such

$$
\left|\sum_{k=1}^{n}\left(1+\frac{q_{k-1}}{q_{k}}\right)-\frac{n}{\log 2}\right|<\lambda n^{1-\delta} .
$$

Lemma 1 is of the same nature as Lemma 4 of [2], and is proved in the same way, taking the quantity $F_{i}\left(a_{i}, \cdots, a_{i-k_{i+1}}\right)$ which occurred in [2] to be

$$
1+\frac{1}{a_{i}+} \frac{1}{a_{i-1}+} \cdots \frac{1}{+a_{i-k_{i}+1}} .
$$

The uniform boundedness of $\int_{0}^{1} F_{i}^{2} d x$ is now trivial, since $1<F_{i}<2$. Equation (5) is now derived by taking $\alpha_{k}=1+q_{k-1} / q_{k}, \alpha=(\log 2)^{-1}$.

We note in passing that equation (5) was used only twice, namely in the proofs of (10) and the equation preceding (11), in [2]. For the application which was made of (10), simple asymptotic equality would have sufficed; 
no estimate of the error term is needed. This is not true of the equation preceding (11).

In the direction of $I^{\prime \prime}$, we have the following theorem.

THEOREM 1. Let $f$ be a decreasing function satisfying conditions (1), and let

$$
\begin{aligned}
& g(x)=\frac{f(\log x)}{x}, \quad G(n)=\sum_{k=1}^{n} g(n), \\
& T_{n}^{(d)}=\operatorname{No}\{m, l|| m x-l \mid<g(m), m \leqq n,(m, l) \leqq d\}
\end{aligned}
$$

where $d$ is a positive integer. Then for fixed $d$,

$$
\begin{aligned}
\lim _{n \rightarrow \infty} \operatorname{Tr}\left\{T_{n}^{(d)}<2\left(1-\frac{6}{\pi^{2}} \sum_{r=d+1}^{\infty} r^{-2}\right)\right. & G(n) \\
& \left.\quad+\omega\left(\left(12 \pi^{-2} \sum_{r=1}^{d} \frac{2 r-1}{r^{2}}\right) G(n)\right)^{1 / 2}\right\}=\phi(\omega) .
\end{aligned}
$$

Notice that the coefficient in the first occurrence of $G(n)$ is very nearly 2 , as in $I^{\prime \prime}$, but that the coefficient in the second occurrence is roughly $c \log d$.

To prove Theorem 1 we use the following lemma.

Lemma 2. Let $d$ and $k$ be positive integers, and suppose that $0<h<1 / 2$. Let $x$ be a random variable, uniformly distributed on $[0,1]$, and put

$$
V_{k}=V_{k}(h)=\left\{\begin{array}{rr}
r-h \zeta_{d}(2)\left(1+q_{k-1} / q_{k}\right) & \text { if } \frac{h}{(r+1)^{2} q_{k}}<\left\langle q_{k} x\right\rangle \leqq \frac{h}{r^{2} q_{k}}, \\
r & =0,1, \cdots, d-1, \\
d-h \zeta_{d}(2)\left(1+q_{k-1} / q_{k}\right) & \text { if }\left\langle q_{k} x\right\rangle \leqq \frac{h}{d^{2} q_{k}},
\end{array}\right.
$$

where $\zeta_{d}(2)=\sum_{1}^{d} m^{-2}$. Then

$$
E_{k}\left(V_{k}\right)=E\left(V_{k} \mid q_{0}, q_{1}, \cdots, q_{k}\right)=0
$$

and

$$
\left|E_{k}\left(V_{k}^{2}\right)-h\left(\sum_{m=1}^{d} \frac{2 m-1}{m^{2}}\right)\left(1+q_{k-1} / q_{k}\right)\right|<c h^{2},
$$

where $c$ is an absolute constant.

Let

$$
\alpha_{k}=h\left(1+\frac{q_{k-1}}{q_{k}}\right), \quad \beta_{k}=\zeta_{d}(2) \alpha_{k}
$$


Then for $r=1,2, \cdots, d-1$,

$$
\begin{aligned}
\operatorname{Pr}_{k}\left\{V_{k}=r-\beta_{k}\right\} & =\operatorname{Pr}_{k}\left\{\frac{h}{(r+1)^{2} q_{k}}<\frac{1}{q_{k} x_{k+1}+q_{k-1}} \leqq \frac{h}{r^{2} q_{k}}\right\} \\
& =\operatorname{Pr}_{k}\left\{\frac{r^{2}}{h}-\frac{q_{k-1}}{q_{k}} \leqq x_{k+1}<\frac{(r+1)^{2}}{h}-\frac{q_{k-1}}{q_{k}}\right\} .
\end{aligned}
$$

Since

$$
1=\frac{1}{1 / 2}-1<\frac{r^{2}}{h}-\frac{q_{k-1}}{q_{k}}=\xi_{k}<\frac{(r+1)^{2}}{h}-\frac{q_{k-1}}{q_{k}}=\eta_{k},
$$

all values in the interval indicated for $x_{k+1}$ can actually be assumed by $x_{k+1}$, so that

$$
\begin{aligned}
\operatorname{Pr}_{k}\left\{V_{k}=r-\beta_{k}\right\} & =\operatorname{Pr}_{k}\left\{x \in\left[\frac{p_{k} \xi_{k}+p_{k-1}}{q_{k} \xi_{k}+q_{k-1}}, \frac{p_{k} \eta_{k}+p_{k-1}}{q_{k} \eta_{k}+q_{k-1}}\right]\right\} \\
& =\frac{\left|\frac{p_{k} \xi_{k}+p_{k-1}}{q_{k} \xi_{k}+q_{k-1}}-\frac{p_{k} \eta_{k}+p_{k-1}}{q_{k} \eta_{k}+q_{k-1}}\right|}{\left|\frac{p_{k}+p_{k-1}}{q_{k}+q_{k-1}}-\frac{p_{k}}{q_{k}}\right|}
\end{aligned}
$$

and a short computation shows that

$$
\operatorname{Pr}_{k}\left\{V_{k}=r-\beta_{k}\right\}=\alpha_{k}\left(\frac{1}{r^{2}}-\frac{1}{(r+1)^{2}}\right), \quad r=1,2, \cdots, d-1 .
$$

Similarly,

$$
\operatorname{Pr}_{k}\left\{V_{k}=d-\beta_{k}\right\}=\operatorname{Pr}_{k}\left\{x_{k+1} \geqq \frac{d^{2}}{h}-\frac{q_{k-1}}{q_{k}}\right\}=\frac{\alpha_{k}}{d^{2}},
$$

and since the total probability is 1 ,

$$
\operatorname{Pr}_{k}\left\{V_{k}=-\beta_{k}\right\}=1-\alpha_{k} .
$$

Hence

$$
\begin{aligned}
E_{k}\left(V_{k}\right)= & -\beta_{k}\left(1-\alpha_{k}\right)+\alpha_{k} \sum_{r=1}^{d-1}\left(\frac{1}{r^{2}}-\frac{1}{(r+1)^{2}}\right)\left(r-\beta_{k}\right)+\left(d-\beta_{k}\right) \frac{\alpha_{k}}{d^{2}} \\
= & -\beta_{k}\left(1-\alpha_{k}\right)+\alpha_{k}\left(1+\frac{1}{2^{2}}+\cdots+\frac{1}{(d-1)^{2}}-\frac{d-1}{d^{2}}\right) \\
& -\alpha_{k} \beta_{k}\left(1-\frac{1}{d^{2}}\right)+\frac{\alpha_{k}}{d}-\frac{\alpha_{k} \beta_{k}}{d^{2}} \\
= & 0
\end{aligned}
$$


and

$$
\begin{aligned}
E_{k}\left(V_{k}^{2}\right)= & \beta_{k}^{2}\left(1-\alpha_{k}\right)+\alpha_{k} \sum_{r=1}^{d-1}\left(\frac{1}{r_{2}}-\frac{1}{(r+1)^{2}}\right)\left(r-\beta_{k}\right)^{2}+\left(d-\beta_{k}\right)^{2} \frac{\alpha_{k}}{d^{2}} \\
= & \beta_{k}^{2}\left(1-\alpha_{k}\right)+\alpha_{k}\left(\sum_{r=1}^{d} \frac{2 r-1}{r^{2}}-1\right)-2 \alpha_{k} \beta_{k}\left(\sum_{r=1}^{d} \frac{1}{r^{2}}-\frac{1}{d}\right) \\
& +\alpha_{k} \beta_{k}^{2}\left(1-\frac{1}{d^{2}}\right)+\left(d-\beta_{k}\right)^{2} \frac{\alpha_{k}}{d^{2}} \\
= & \alpha_{k} \sum_{r=1}^{d} \frac{2 r-1}{r^{2}}+\beta_{k}^{2}-2 \alpha_{k} \beta_{k} \zeta_{d}(2) \\
= & \alpha_{k} \sum_{r=1}^{d} \frac{2 r-1}{r^{2}}-\beta_{k}^{2},
\end{aligned}
$$

so that (6) holds with $c=\left(2 \cdot \pi^{2} / 6\right)^{2}$.

Continuing with the proof of Theorem 1, consider the quantity

$$
W_{n}^{(d)}=\text { No }\left\{k, r \mid\left\langle q_{k} x\right\rangle<\frac{f(k)}{r^{2} q_{k}}, r q_{k} \leqq q_{n}, 1 \leqq r \leqq d\right\} ;
$$

the difference between this and

$$
\sum_{k=1}^{n}\left(V_{k}(f(k))+\beta_{k}\right)=\text { No }\left\{k, r \mid\left\langle q_{k} x\right\rangle<\frac{f(k)}{r^{2} q_{k}}, k \leqq n, 1 \leqq r \leqq d\right\}
$$

is just

$$
\gamma=\text { No }\left\{k, r \mid\left\langle q_{k} x\right\rangle<\frac{f(k)}{r^{2} q_{k}}, k \leqq n, 1 \leqq r \leqq d, r q_{k}>q_{n}\right\} .
$$

Since $q_{n} \geqq q_{n-1}+q_{n-2} \geqq 2 q_{n-2}$, we have

$$
\frac{q_{n}}{q_{k}} \geqq 2^{[(n-k) / 2]} \quad \text { for } n \geqq k .
$$

Hence

$$
\begin{aligned}
\gamma & \leqq \text { No }\left\{k, r \mid k \leqq n, 1 \leqq r \leqq d, r q_{k}>q_{n}\right\} \leqq d \cdot \text { No }\left\{k \mid k \leqq n, q_{n} / q_{k}<d\right\} \\
& \leqq d \cdot \text { No }\left\{k \leqq n \mid 2^{[(n-k) / 2]}<d\right\}<d^{2},
\end{aligned}
$$

and since $d$ is fixed,

$$
W_{n}^{(d)}=\sum_{k=1}^{n} V_{k}(f(k))+\sum_{k=1}^{n} \beta_{k}+O(1),
$$

as $n \rightarrow \infty$. Using the definition of $\beta_{k}$ (with $h=f(k)$ ) and (5), we see that for almost all $x$, 


$$
W_{n}^{(d)}=\sum_{k=1}^{n} V_{k}(f(k))+\frac{\zeta_{d}(2)}{\log 2} \sum_{k=1}^{n} f(k)+O(1) .
$$

From (5) and Lemma 2 we have, for almost all $x$,

$$
\sum_{k=1}^{n} E_{k}\left(V_{k}^{2}(f(k))\right) \sim \frac{1}{\log 2} \sum_{m=1}^{d} \frac{2 m-1}{m^{2}} \sum_{k=1}^{n} f(k) .
$$

Hence by Lemma 3 of [2],

(7) $\lim _{n \rightarrow \infty} \operatorname{Pr}\left\{W_{n}^{(d)}<\frac{\zeta_{d}(2)}{\log 2} \sum_{k=1}^{n} f(k)+\omega\left(\frac{1}{\log 2} \sum_{m=1}^{d} \frac{2 m-1}{m^{2}} \sum_{k=1}^{n} f(k)\right)^{1 / 2}\right\}=\phi(\omega)$.

Equation (7) is an extension of Lemma 2 of [2], and reduces to it in case $d=1$. The remainder of the proof of Theorem 1 is exactly parallel to that of Theorem 1 of [2], requiring only trivial modifications, and we omit it.

3. Some lemmas. In the next section we shall be concerned with a function $f$ satisfying the following conditions:

$f$ is a nonincreasing function on the positive real numbers,

$$
\begin{aligned}
& 0<f(x)<1 / 2 \text { for } x>0, \\
& \sum_{k=1}^{\infty} f(k) \text { diverges, so that } F(n)=\sum_{k=1}^{n} f(k) \rightarrow \infty \text { as } n \rightarrow \infty .
\end{aligned}
$$

We shall need the following results.

LEMмa 3. If $f$ satisfies conditions (8), and $\left\{b_{k}\right\}$ is a bounded sequence such that $B_{n}=\sum_{1}^{n} b_{k}=O\left(n^{\delta}\right)$ for some fixed $\delta<1$, then

$$
\sum_{k=1}^{n} b_{k} f(k)=o(F(n))
$$

Since $\sum f(k)$ diverges, it is possible to choose a sequence $\left\{h_{n}\right\}$ of positive integers tending to $\infty$ in such a way that $F\left(h_{n}\right)=o(F(n))$. We write

$$
\begin{aligned}
\sum_{k=1}^{n} b_{k} f(k) & =\sum_{k=1}^{h_{n}} b_{k} f(k)+\sum_{k=h_{n}+1}^{n}\left(B_{k}-B_{k-1}\right) f(k) \\
& =\sum_{k=1}^{h_{n}} b_{k} f(k)+\sum_{k=h_{n}+1}^{n-1} \frac{B_{k}}{k}(k f(k)-k f(k+1))+B_{n} f(n)-B_{h_{n}} f\left(h_{n}+1\right),
\end{aligned}
$$

so that

$$
\begin{aligned}
\left|\sum_{k=1}^{n} b_{k} f(k)\right| \leqq & M \sum_{k=1}^{h_{n}} f(k)+\sum_{k=h_{n}+1}^{n-1} \frac{c k^{\delta}}{k}|k f(k)-k f(k+1)| \\
& +c n^{\delta} f(n)+c h_{n}^{\delta} f\left(h_{n}\right) .
\end{aligned}
$$


Now the second sum on the right is at most

$$
\begin{aligned}
\sum_{k=h_{n}+1}^{n-1} \frac{c}{h_{n}^{1-\delta}}|k f(k)-k f(k+1)| & =\frac{c}{h_{n}^{1-\delta}} \sum_{k=h_{n}+1}^{n-1}(k f(k)-k f(k+1)) \\
& =\frac{c}{h_{n}^{1-\delta}}\left(\sum_{k=h_{n}+1}^{n} f(k)+h_{n} f\left(h_{n}+1\right)-n f(n)\right),
\end{aligned}
$$

so that

$$
\begin{aligned}
\left|\sum_{k=1}^{n} b_{k} f(k)\right| \leqq & M F\left(h_{n}\right)+\frac{c}{h_{n}^{1-\delta}} F(n)+2 c h_{n}^{\delta} f\left(h_{n}\right) \\
& +c\left(\frac{1}{n^{1-\delta}}+\frac{1}{h_{n}^{1-\delta}}\right) n f(n) .
\end{aligned}
$$

By the monotonicity of $f, F(n) \geqq n f(n)$, and hence $n^{\delta} f(n) \leqq n^{\delta-1} F(n)$. Thus all four terms on the right hand side of $(9)$ are $o(F(n))$.

Lemma 4. If $f$ satisfies conditions (8), and $\alpha$ and $\delta$ are constants with $\delta<1$, then

$$
\sum_{k=1}^{n} f\left(k-\alpha k^{\delta}\right) \sim F(n) \text { as } n \rightarrow \infty
$$

We consider the case $\alpha>0$; the proof when $\alpha<0$ is similar. Put

and write

$$
\begin{aligned}
\Delta(k) & =\left((k+1)-\alpha(k+1)^{\delta}\right)-\left(k-\alpha k^{\delta}\right) \\
& =1-\alpha k^{\delta}\left\{\left(1+\frac{1}{k}\right)^{\delta}-1\right\} \\
& =1+O\left(k^{\delta-1}\right),
\end{aligned}
$$

$$
\begin{aligned}
F^{*}(n) & =\sum_{k=1}^{n} f\left(k-\alpha k^{\delta}\right) \\
& =\sum_{k=1}^{n} f\left(k-\alpha k^{\delta}\right) \Delta(k)+O\left(\sum_{k=1}^{n} k^{\delta-1} f\left(k-\alpha k^{\delta}\right)\right) .
\end{aligned}
$$

Since $f$ is a nonincreasing function,

$$
\sum_{k=1}^{n} f\left(k-\alpha k^{\delta}\right) \Delta(k)=\int_{1}^{n} f\left(t-\alpha t^{\delta}\right) d t+O(1) ;
$$

putting $t-\alpha t^{\delta}=u$, we obtain 


$$
\begin{aligned}
\int_{1}^{n} f\left(t-\alpha t^{\delta}\right) d t & =\int_{1}^{n-\alpha n^{\delta}} f(u) \frac{d u}{1-\alpha \delta t^{\delta-1}}+O(1) \\
& \leqq \int_{1}^{n} f(u) \frac{d u}{1-\alpha \delta u^{\delta-1}}+O(1) \\
& \leqq \int_{1}^{n} f(u)\left(1+2 \alpha \delta u^{\delta-1}\right) d u+O(1) \\
& =F(n)+O\left(\sum_{k=1}^{n} k^{\delta-1} f(k)\right)
\end{aligned}
$$

so that

$$
F(n) \leqq F^{*}(n)
$$

$$
\leqq F(n)+O\left(\sum_{k=1}^{n} k^{\delta-1} f\left(k-\alpha k^{\delta}\right)\right) .
$$

If the sum in the last written error term converges as $n \rightarrow \infty$, the proof is complete. Otherwise, write

$$
\begin{aligned}
\sum_{k=1}^{n} k^{\delta-1} f(k- & \left.\alpha k^{\delta}\right) \\
& =\sum_{k=1}^{h_{n}} k^{\delta-1} f\left(k-\alpha k^{\delta}\right)+\sum_{k=h_{n}+1}^{n-1} F^{*}(k)\left(k^{\delta-1}-(k+1)^{\delta-1}\right)+\frac{F^{*}(n)}{n^{1-\delta}} \\
& \leqq \sum_{k=1}^{h_{n}} k^{\delta-1} f\left(k-\alpha k^{\delta}\right)+F^{*}(n)\left(\frac{1}{\left(h_{n}+1\right)^{1-\delta}}-\frac{1}{n^{1-\delta}}\right)+\frac{F^{*}(n)}{n^{1-\delta}}
\end{aligned}
$$

and choose $\left\{h_{n}\right\}$ as a sequence going to infinity in such a way that

$$
\sum_{k=1}^{h_{n}} k^{\delta-1} f\left(k-\alpha k^{\delta}\right)=o\left(\sum_{k=1}^{n} k^{\delta-1} f\left(k-\alpha k^{\delta}\right)\right) .
$$

Then

$$
\{1-o(1)\} \sum_{k=1}^{n} k^{\delta-1} f\left(k-\alpha k^{\delta}\right)=o\left(F^{*}(n)\right),
$$

and the lemma follows from (10).

Corollary. Under the same hypotheses,

$$
\sum_{k=1}^{n-\alpha n^{\delta}} f(k) \sim F(n) \text { as } n \rightarrow \infty .
$$

For 


$$
\begin{aligned}
\sum_{k=1}^{n-\alpha n^{\delta}} f(k) & =\int_{1}^{n-\alpha n^{\delta}} f(t) d t+O(1) \\
& =\int_{1}^{n} f\left(u-\alpha u^{\delta}\right)\left(1-\alpha \delta u^{\delta-1}\right) d u+O(1) \\
& =(1+o(1)) \int_{1}^{n} f\left(u-\alpha u^{\delta}\right) d u=(1+o(1)) F^{*}(n) \sim F(n) .
\end{aligned}
$$

4. The Main Theorem. In the present section we shall prove this theorem:

THEOREM 2. Suppose that $\mathrm{g}$ is a function satisfying the following conditions:

(a) $x g(x)$ is nonincreasing, and $0<x g(x)<1 / 2$, for $x \geqq 0$,

(b) $x g(x) \rightarrow 0$ as $x \rightarrow \infty$,

(c) $\sum_{k=1}^{\infty} g(k)$ diverges.

Then for almost all $x$, the number of solutions $m \leqq n$ of the inequality

$$
\langle m x\rangle<g(m)
$$

is asymptotic to $2 \sum_{1}^{n} g(k)$.

It is easily seen that for $f(x)=e^{x} g\left(e^{x}\right)$, the conditions (a) and (c) of (11) are equivalent to conditions (8), and that the three conditions (11) are equivalent to

$$
\begin{aligned}
& \text { conditions (8) hold, } \\
& f(x) \rightarrow 0 \text { as } x \rightarrow \infty .
\end{aligned}
$$

I conjecture $\left({ }^{1}\right)$ that Theorem 2 remains true if condition (b) of (11) is omitted; it has therefore seemed useful to prove the results of the preceding section without using (b). At any rate, since the conditions (12) are considerably weaker than those in (1), Theorem 2 is stronger than the assertion $\mathrm{II}^{\prime \prime}$ of the introduction.

The following lemma is the first step in the proof of Theorem 2.

LemMa 5. Let $f$ be a function satisfying (12), and let $B$ be a non-negative constant. Let $q_{0}, q_{1}, \cdots$ be the denominators of the convergents to the real number $x$, and let

$$
S_{n}=S_{n}(x)=\text { No }\left\{k, r \mid\left\langle q_{k} x\right\rangle<\frac{f(k+B \log r)}{r^{2} q_{k}}, 1 \leqq r q_{k} \leqq q_{n}\right\} \text {. }
$$

(1) Note added in proof. This conjecture has since been proved by Erdös; the proof will appear in Acta Arithmetica. 
Then for almost all $x$,

$$
S_{n}(x) \sim \frac{\pi^{2}}{6 \log 2} F(n) \text { as } n \rightarrow \infty .
$$

Instead of $S_{n}$, we first consider the quantity

$$
R_{n}=\text { No }\left\{k, r \mid\left\langle q_{k} x\right\rangle<\frac{f(k+B \log r)}{r^{2} q_{k}}, 1 \leqq r \leqq \phi_{k}, k \leqq n\right\},
$$

where $\left\{\phi_{k}\right\}$ is an unbounded nondecreasing sequence of positive integers which will be determined later so as to satisfy certain conditions. It would seem at first sight that the behavior of $R_{n}$, for sufficiently slowly growing $\left\{\phi_{k}\right\}$, could be analyzed directly with the help of Lemma 2, in the same way in which (7) was established. This is not the case, however, for the following reason. As was pointed out in the appended note in [2], the proofs of assertions $\mathrm{I}^{\prime}$ and $\mathrm{II}^{\prime}$ ' of the introduction depend on limit theorems proved by Lévy, of the form, "If $Z_{1}, Z_{2}, \cdots$ is a sequence of random variables such that the conditional expectation $E\left(Z_{\nu} \mid Z_{1}, \cdots, Z_{\nu-1}\right)$ of $Z_{\nu}$, given $Z_{1}, \cdots, Z_{\nu-1}$, is zero for $\nu=1,2, \cdots$, then $\cdots$." In Lemma 2 it is verified only that $E\left(V_{k} \mid q_{0}, \cdots, q_{k}\right)=0$, and to apply Lévy's theorems it would have to be true that the numbers $q_{0}, \cdots, q_{k}$ uniquely determine the values of $V_{0}, \cdots, V_{k-1}$. But this is sometimes false. For let $q_{0}, \cdots, q_{k}$ be fixed; then to determine $V_{k-1}$ we must (in some cases, at least) find the integer $r_{k-1}$ such that

$$
\frac{h_{k-1}}{\left(r_{k-1}+1\right)^{2} q_{k-1}}<\left\langle q_{k-1} x\right\rangle \leqq \frac{h_{k-1}}{r_{k-1}^{2} q_{k}} .
$$

Now

$$
\left\langle q_{k-1} x\right\rangle=\frac{1}{q_{k}+\frac{q_{k-1}}{x_{k+1}}},
$$

so that

$$
r_{k-1}=\left[\left(\frac{h_{k-1}}{q_{k-1}\left\langle q_{k-1} x\right\rangle}\right)^{1 / 2}\right]=\left[\left(h_{k-1}\left(\frac{q_{k}}{q_{k-1}}+\frac{1}{x_{k+1}}\right)\right)^{1 / 2}\right],
$$

and if there is an integer in the interval between $\left(h_{k-1} q_{k} / q_{k-1}\right)^{1 / 2}$ and $\left(h_{k-1}\left(q_{k} / q_{k-1}+1\right)\right)^{1 / 2}$, then there are two different values of $x$ with the same $q_{0}, \cdots, q_{k}$ but corresponding to different values of $r_{k-1}$.

In [2] this difficulty was surmounted by noticing that under the stringent hypotheses (1) concerning the function $f$ which were then in force, all but a finite number of the $r_{k}$ are uniquely determined by the appropriate $q$ 's, for 
almost all $x$. (Roughly speaking, if $f(k-1)=h_{k-1}$ is sufficiently small, then it is very unlikely that there is an integer in the above mentioned interval.) This device is no longer available, so we proceed somewhat differently.

Let $\log u=0$ for $0 \leqq u \leqq 1$, and $=\log u$ for $u>1$. For $k \geqq 1$, let $r_{k}$ be the nonnegative integer determined by the inequality

$$
\frac{f\left(k+B \log \left(\boldsymbol{r}_{k}+1\right)\right)}{\left(\boldsymbol{r}_{k}+1\right)^{2} q_{k}}<\left\langle q_{k} x\right\rangle \leqq \frac{f\left(k+B \log \boldsymbol{r}_{k}\right)}{\boldsymbol{r}_{k}^{2} q_{k}} .
$$

Let

$$
Q_{k}= \begin{cases}r_{k} & \text { if } r_{k}<\phi_{k} \\ \phi_{k} & \text { otherwise }\end{cases}
$$

so that

$$
R_{n}=\sum_{k=1}^{n} Q_{k}
$$

For brevity set $\bar{k}=k+B \log \phi_{k}$, and put

$$
Q_{k}^{\prime}= \begin{cases}\left.\left[f(\bar{k}) q_{k+1} / q_{k}\right)^{1 / 2}\right] & \text { if this quantity is smaller than } \phi_{k}, \\ \phi_{k} & \text { otherwise }\end{cases}
$$

and

$$
Q_{k}^{\prime \prime}=\left\{\begin{array}{l}
{\left[\left(f(k)\left(\frac{q_{k+1}}{q_{k}}+1\right)\right)^{1 / 2}\right] \text { if this quantity is smaller than } \phi_{k},} \\
\phi_{k} \text { otherwise. }
\end{array}\right.
$$

We have, in analogy with (13),

$$
\begin{gathered}
r_{k}^{2} \leqq f\left(k+B \log r_{k}\right)\left(\frac{q_{k+1}}{q_{k}}+\frac{1}{x_{k+1}}\right), \\
\left(r_{k}+1\right)^{2} \geqq f\left(k+B \log \left(r_{k}+1\right)\right)\left(\frac{q_{k+1}}{q_{k}}+\frac{1}{x_{k+1}}\right) .
\end{gathered}
$$

By the first of these inequalities, $Q_{k} \leqq Q_{k}^{\prime \prime}$. From the second we see that when $r_{k}<\phi_{k}, Q_{k} \geqq Q_{k}^{\prime}$. When $r_{k} \geqq \phi_{k}, Q_{k}=\phi_{k}$, while $Q_{k}^{\prime} \leqq \phi_{k}$ always, so in all cases,

$$
Q_{k}^{\prime} \leqq Q_{k} \leqq Q_{k}^{\prime \prime} \text {. }
$$

From the nature of their definitions, $Q_{0}^{\prime}, \cdots, Q_{k-1}^{\prime}$, as well as $Q_{0}^{\prime \prime}, \cdots, Q_{k-1}^{\prime \prime}$, are uniquely determined by $q_{0}, \cdots, q_{k}$. From (14) and (15), any common asymptotic estimate for $\sum_{1}^{n} Q_{k}^{\prime}$ and $\sum_{1}^{n} Q_{k}^{\prime \prime}$ is also an asymptotic estimate for $R_{n}$. We shall prove that for almost all $x$, 


$$
\sum_{k=1}^{n} Q_{k}^{\prime} \sim \frac{\pi^{2}}{6 \log 2} F(n) \quad \text { and } \quad \sum_{k=1}^{n} Q_{k}^{\prime \prime} \sim \frac{\pi^{2}}{6 \log 2} F(n)
$$

if $f$ satisfies (12), and the $\phi_{k}$ are suitably chosen. We give details only for $\sum_{1}^{n} Q_{k}^{\prime}$, the other sum being treated similarly.

As before, let $\operatorname{Pr}_{k}$ and $\mathrm{E}_{k}$ denote conditional probability and expectation, given $q_{0}, \cdots, q_{k}$, the basic random variable being $x$, uniformly distributed on $[0,1]$. For brevity put

$$
r_{k}^{\prime}=\left[\left(\frac{f(\bar{k}) q_{k+1}}{q_{k}}\right)^{1 / 2}\right]
$$

Then $r_{k}^{\prime}=r$ if and only if

$$
\frac{r^{2} q_{k}}{f(\bar{k})} \leqq q_{k+1}<\frac{(r+1)^{2} q_{k}}{f(\bar{k})} ;
$$

since $q_{k+1}=q_{k}\left[x_{k+1}\right]+q_{k-1}$, we have that $r_{k}^{\prime}=r$ exactly when

$$
\frac{r^{2}}{f(\bar{k})}-\frac{q_{k-1}}{q_{k}} \leqq\left[x_{k+1}\right]<\frac{(r+1)^{2}}{f(\bar{k})}-\frac{q_{k-1}}{q_{k}},
$$

whence it follows that if $r<\phi_{k}$,

$$
\operatorname{Pr}_{k}\left\{Q_{k}^{\prime}=r\right\} \leqq \operatorname{Pr}_{k}\left\{\frac{r^{2}}{f(\bar{k})}-\frac{q_{k-1}}{q_{k}} \leqq x_{k+1}<\frac{(r+1)^{2}}{f(\bar{k})}-\frac{q_{k-1}}{q_{k}}+1\right\},
$$

and

$$
\operatorname{Pr}_{k}\left\{Q_{k}^{\prime}=r\right\} \geqq \operatorname{Pr}_{k}\left\{\frac{r^{2}}{f(\bar{k})}-\frac{q_{k-1}}{q_{k}}+1 \leqq x_{k+1}<\frac{(r+1)^{2}}{f(\bar{k})}-\frac{q_{k-1}}{q_{k}}\right\} .
$$

By the same kind of computation as was carried out in the proof of Lemma 2, we find that for $0<r<\phi_{k}$,

$$
\bar{\alpha}_{k}\left(\frac{1}{r^{2}+f(\bar{k})}-\frac{1}{(r+1)^{2}}\right) \leqq \operatorname{Pr}_{k}\left\{Q_{k}^{\prime}=r\right\} \leqq \bar{\alpha}_{k}\left(\frac{1}{r^{2}}-\frac{1}{(r+1)^{2}+f(\bar{k})}\right),
$$

where

$$
\bar{\alpha}_{k}=f(\bar{k})\left(1+\frac{q_{k-1}}{q_{k}}\right) .
$$

Similarly,

$$
\frac{\bar{\alpha}_{k}}{\phi_{k}^{2}+f(\bar{k})} \leqq \operatorname{Pr}_{k}\left\{Q_{k}^{\prime}=\phi_{k}\right\} \leqq \frac{\bar{\alpha}_{k}}{\phi_{k}^{2}},
$$

and $\operatorname{Pr}_{k}\left\{Q_{k}^{\prime}=0\right\}$ is well defined. Hence 


$$
\begin{aligned}
\mathrm{E}_{k}\left(Q_{k}^{\prime}\right)= & \sum_{r=0}^{\phi_{k}} r \operatorname{Pr}_{k}\left\{Q_{k}^{\prime}=r\right\} \leqq \bar{\alpha}_{k} \sum_{r=1}^{\phi_{k}-1} r\left(\frac{1}{r^{2}}-\frac{1}{(r+1)^{2}+f(\bar{k})}\right)+\frac{\bar{\alpha}_{k}}{\phi_{k}^{2}} \cdot \phi_{k} \\
= & \bar{\alpha}_{k} \sum_{r=1}^{\phi_{k}-1} r\left(\frac{1}{r^{2}}-\frac{1}{(r+1)^{2}}\right)+\bar{\alpha}_{k} \sum_{r=1}^{\phi_{k}-1} r\left(\frac{1}{(r+1)^{2}}-\frac{1}{(r+1)^{2}+f(\bar{k})}\right) \\
& +\frac{\bar{\alpha}_{k}}{\phi_{k}} \\
= & \bar{\alpha}_{k}\left(\zeta_{\phi_{k}}(2)-\frac{1}{\phi_{k}}\right)+\bar{\alpha}_{k} f(k) \sum_{r=1}^{\phi_{k}-1} \frac{r}{(r+1)^{2}\left((r+1)^{2}+f(\bar{k})\right)}+\frac{\bar{\alpha}_{k}}{\phi_{k}} \\
= & \bar{\alpha}_{k} \zeta_{\phi_{k}}(2)+O\left(\bar{\alpha}_{k} f(\bar{k})\right),
\end{aligned}
$$

and in the same way,

$$
\begin{aligned}
\mathrm{E}_{k}\left(Q_{k}^{\prime}\right) & \geqq \bar{\alpha}_{k} \zeta_{\phi_{k}}(2)+O\left(\bar{\alpha}_{k} f(\bar{k})\right), \\
\mathrm{E}_{k}\left(Q_{k}^{\prime 2}\right) & =2 \bar{\alpha}_{k} \log \phi_{k}+O\left(\bar{\alpha}_{k}\right) .
\end{aligned}
$$

Hence there is a sequence of constants $\bar{\beta}_{k}$ such that

$$
\begin{aligned}
& \bar{\beta}_{k}= \bar{\alpha}_{k} \zeta_{\phi_{k}}(2)+O\left(\bar{\alpha}_{k} f(\bar{k})\right), \mathrm{E}_{k}\left(Q_{k}^{\prime}-\bar{\beta}_{k}\right)=0, \\
& \operatorname{Var}_{k}\left(Q_{k}^{\prime}-\bar{\beta}_{k}\right)=\mathrm{E}_{k}\left(\left(Q_{k}^{\prime}-\bar{\beta}_{k}\right)^{2}\right)=2 \bar{\alpha}_{k} \log \phi_{k}+O\left(\alpha_{k}\right) .
\end{aligned}
$$

Since $1<1+q_{k-1} / q_{k}<2$, we find that

$$
\begin{aligned}
2 \sum_{k=1}^{n} f(\bar{k}) \log \phi_{k}+ & O(\bar{F}(n)) \\
& \leqq \sum_{k=1}^{n} \operatorname{Var}_{k}\left(Q_{k}^{\prime}-\bar{\beta}_{k}\right) \leqq 4 \sum_{k=1}^{n} f(\bar{k}) \log \phi_{k}+O(\bar{F}(n)),
\end{aligned}
$$

where

$$
r(n)=\sum_{k=1}^{n} f(\bar{k})
$$

We now suppose that the sequence $\phi_{1}, \phi_{2}, \ldots$ is chosen in such a way that

$$
\sum_{k=1}^{n} f(k) \log \phi_{k}=O\left(F^{2-\bullet}(n)\right)
$$

for some positive constant $\epsilon$, and

$$
\phi_{k}=O(k) .
$$

Then by (18) and (19), 


$$
\sum_{k=1}^{n} \operatorname{Var}_{k}\left(Q_{k}^{\prime}-\bar{\beta}_{k}\right)=O\left(F^{2-\epsilon}(n)\right) .
$$

Lévy $\left[3\right.$, p. 253, formula $\left.\left(23^{\prime}\right)\right]$ has shown that if $Z_{1}, Z_{2}, \cdots$ is a sequence of random variables such that $\mathrm{E}\left(Z_{k} \mid Z_{1}, \cdots, Z_{k-1}\right)=0$, and if $\sum \operatorname{Var}\left(Z_{k} \mid Z_{1}, \cdots, Z_{k-1}\right)$ diverges with probability 1 , then if $\epsilon^{\prime}>0$ there is almost surely a number $t^{\prime}$ such that

$$
\begin{aligned}
\left|\sum_{k=1}^{n} Z_{k}\right|< & \left(\sum_{k=1}^{n} \operatorname{Var}\left(Z_{k} \mid Z_{1}, \cdots, Z_{k-1}\right)\right)^{1 / 2+\epsilon^{\prime}} \\
& \text { for all } n \text { such that } \sum_{k=1}^{n} \operatorname{Var}\left(Z_{k} \mid Z_{1}, \cdots, Z_{k-1}\right)>t^{\prime} .
\end{aligned}
$$

(For a purpose irrelevant here, Lévy assumed also that the $Z_{k}$ are uniformly bounded, but this hypothesis is not used in the proof of the theorem just stated.) We apply Lévy's result in the case $Z_{k}=Q_{k}^{\prime}-\bar{\beta}_{k}, \epsilon^{\prime}=\epsilon / 4$; by (8), (17) and the first inequality of (18), the hypotheses are satisfied, and by (21) and (22),

$$
\sum_{k=1}^{n}\left(Q_{k}^{\prime}-\bar{\beta}_{k}\right)=O\left(F^{\left(1 / 2+\varepsilon^{\prime}\right)(2-\epsilon)}(n)\right)=o(F(n)) \quad \text { for almost all } x .
$$

Finally,

$$
\begin{aligned}
\sum_{k=1}^{n} \bar{\beta}_{k} & =\sum_{k=1}^{n} f(\bar{k}) \zeta_{\phi_{k}}(2)\left(1+\frac{q_{k-1}}{q_{k}}\right)+O\left(\sum_{k=1}^{n} f^{2}(\bar{k})\left(1+\frac{q_{k-1}}{q_{k}}\right)\right) \\
& =\frac{\pi^{2}}{6} \sum_{k=1}^{n} f(\bar{k})\left(1+\frac{q_{k-1}}{q_{k}}\right)+O\left(\sum_{k=1}^{n} \frac{f(\bar{k})}{\phi_{k}}\right)+O\left(\sum_{k=1}^{n} f^{2}(\bar{k})\right),
\end{aligned}
$$

and by (11b) and the fact that $\phi_{k} \rightarrow \infty$ as $k \rightarrow \infty$,

$$
\sum_{k=1}^{n} \bar{\beta}_{k}=\frac{\pi^{2}}{6} \sum_{k=1}^{n} f(\bar{k})\left(1+\frac{q_{k-1}}{q_{k}}\right)+o(F(n)) .
$$

(This is the only direct application of condition (11b)). By (20) and Lemmas 1 and 3,

$$
\sum_{k=1}^{n} \bar{\beta}_{k} \sim \frac{\pi^{2}}{6} \frac{1}{\log 2} F(n) \quad \text { for almost all } x,
$$

and hence by (23)

$$
\sum_{k=1}^{n} Q_{k}^{\prime} \sim \frac{\pi^{2}}{6 \log 2} F(n) \quad \text { for almost all } x .
$$

The proof of the other relation in (16), again assuming (19) and (20), requires no new ideas. 
1960]

By (14), (15) and (16), we have that if (19) and (20) hold then

$$
R_{n} \sim \frac{\pi^{2}}{6 \log 2} F(n) \quad \text { for almost all } x .
$$

We wish to deduce the same asymptotic estimate for $S_{n}$. If we put

$$
M_{n}=\text { No }\left\{k, r \mid\left\langle q_{k} x\right\rangle<\frac{f(k+B \log r)}{r^{2} q_{k}}, r q_{k} \leqq q_{n}, r>\phi_{k}\right\}
$$

and

$$
N_{n}=\text { No }\left\{k, r \mid\left\langle q_{k} x\right\rangle<\frac{f(k+B \log r)}{r^{2} q_{k}}, 1 \leqq r \leqq \phi_{k}, k \leqq n, r q_{k}>q_{n}\right\},
$$

then clearly

$$
S_{n}=R_{n}+M_{n}-N_{n}
$$

Take $0<\delta<1$; then

$$
M_{n} \leqq \text { No }\left\{k, r \mid\left\langle q_{k} x\right\rangle<\frac{f(k) / \phi_{k}^{1+\delta}}{r^{1-\delta} q_{k}}, r q_{k} \leqq q_{n}, r>\phi_{k}\right\} .
$$

If we suppose that

$$
\sum_{k=1}^{\infty} \frac{f(k)}{\phi_{k}^{1+\delta}}<\infty,
$$

then for almost every $x$ there is a $K=K(x)$ such that the inequality

$$
\left\langle q_{k} x\right\rangle<\frac{f(k) / \phi_{k}^{1+8}}{q_{k}}
$$

has no solution with $k \geqq K$. Hence for $n \geqq K$,

$$
\begin{aligned}
M_{n} & \leqq N o\left\{k, r \mid\left\langle q_{k} x\right\rangle<\frac{f(k) / \phi_{k}^{1+\delta}}{r^{1-\delta} q_{k}}, k \leqq K, r q_{k} \leqq q_{n}, r>\phi_{k}\right\} \\
& \leqq N o\left\{k, r \mid\left\langle q_{k} x\right\rangle<\frac{f(k) / \phi_{k}^{1+\delta}}{r^{1-\delta} q_{k}}, k \leqq K, r \geqq 1\right\},
\end{aligned}
$$

and this is a finite number independent of $n$. Hence for almost all $x$,

$$
M_{n}=o\left(R_{n}\right)
$$

if (25) holds.

Now consider $N_{n}$. Since $\phi_{1}, \phi_{2}, \cdots$ is a nondecreasing sequence, 


$$
\mathrm{N}_{n} \leqq \text { No }\left\{k, r \mid\left\langle q_{k} x\right\rangle\left\langle\frac{f(k)}{r^{2} q_{k}}, 1 \leqq r \leqq \phi_{n}, k \leqq n, r q_{k}>q_{n}\right\} .\right.
$$

The inequalities

$$
r \leqq \phi_{n}, \quad k \leqq n, \quad r q_{k}>q_{n}
$$

imply that $q_{k}>q_{n} / \phi_{n}$, or

$$
\log q_{n}-\log q_{k}<\log \phi_{n} .
$$

This implies that $k \rightarrow \infty$ as $n \rightarrow \infty$, because of (20). Also, by Lemma 4 of [2], if $1 / 2<\eta<1$ then for every $x$ not in a certain set of measure 0 , there is a constant $\kappa=\kappa(x)$ such that for all $k$,

$$
\left|\log q_{k}-\frac{\pi^{2}}{12 \log 2} k\right|<\kappa k^{n}
$$

For such $x$ and for $k \leqq n$,

$$
\log q_{n}-\log q_{k}>c(n-k)+O\left(n^{\eta}\right)>c^{\prime}(n-k)
$$

for suitable positive numbers $c$ and $c^{\prime}$, and for $n$ sufficiently large. Hence by (27), for almost every $x$ and for $n>n_{0}(x)$,

$$
c^{\prime}(n-k)<\log \phi_{n}
$$

and there are only $\left[c^{\prime-1} \log \phi_{n}\right]$ solutions $k \leqq n$ of this inequality. Hence

$$
\begin{aligned}
N_{n} & \leqq N o\left\{k, r \mid 1 \leqq r \leqq \phi_{n}, k \leqq n, r q_{k}>q_{n}\right\} \\
& <c^{\prime \prime} \phi_{n} \log \phi_{n},
\end{aligned}
$$

so that if we suppose that

$$
\phi_{n} \log \phi_{n}=o(F(n)),
$$

then

$$
N_{n}=o\left(R_{n}\right) \text {. }
$$

By (24), (26) and (30), it follows that for almost all $x$

$$
S_{n} \sim R_{n} \sim \frac{\pi^{2}}{6 \log 2} F(n) .
$$

The proof of Lemma 5 will therefore be complete if we can find a nondecreasing sequence of positive integers $\phi_{1}, \phi_{2}, \ldots$ satisfying conditions (19), (20), (25), (29). We choose $\phi_{k}=\max \left(1,\left[F^{1-8 / 2}(k)\right]\right)$, with $0<\delta<1$. Then (29) is obvious, (20) follows from the fact that $f(k)=O(1)$, and (25) is a well known theorem due to Abel $[1$, p. 290]. As for (19), we have 


$$
\begin{aligned}
\sum_{k=1}^{n} f(k) \log \phi_{k} & \leqq \log \phi_{n} \sum_{k=1}^{n} f(k)<\log \phi_{n}\left(\phi_{n}+1\right)^{(1-\delta / 2)^{-1}}<\log \phi_{n}\left(\phi_{n}+1\right)^{1+\delta} \\
& <\left(1-\frac{\delta}{2}\right) \log F(n)\left(1+F^{1-\delta / 2}(n)\right)^{1+\delta}
\end{aligned}
$$

and this is smaller than $F^{1+\delta / 2}(n)$ for $n$ sufficiently large. Thus (19) holds if $\epsilon<1 / 2$. This ends the proof of Lemma 5 .

The deduction of Theorem 2 from Lemma 5 proceeds along the same lines as the corresponding part of the proof of assertion $\mathrm{I}^{\prime}$ of the introduction, but is somewhat simpler.

Let $\eta$ be fixed, with $1 / 2<\eta<1$, and for brevity put $B_{0}=\pi^{2} /(12 \log 2)$. Define

$$
\begin{gathered}
s_{n}=s_{n}(x)=\text { No }\left\{k, r \mid\left\langle q_{k} x\right\rangle<\frac{f\left(B_{0}^{-1} \log r q_{k}\right)}{r^{2} q_{k}}, 1 \leqq r q_{k} \leqq q_{n}\right\}, \\
t_{n}(\kappa)=t_{n}(\kappa, x)=\text { No }\left\{k, r \mid\left\langle q_{k} x\right\rangle<\frac{f\left(k-\kappa k^{n}+B_{0}^{-1} \log r\right)}{r^{2} q_{k}}, 1 \leqq r q_{k} \leqq q_{n}\right\} .
\end{gathered}
$$

By Lemmas 4 and 5,

$$
t_{n}(\kappa, x) \sim 2 B_{0} F(n) \text { for each fixed } \kappa, \text { for almost all } x .
$$

Let

$$
G_{k}=\left\{x \in[0,1]|| \log q_{k}-B_{0} k \mid<\kappa k^{n} \text { for every } k \geqq 1\right\},
$$

and let $\delta$ be positive. By (28) we can choose $x$ so large that $\mathfrak{M} G_{k}>1-\delta$. For $x \in G_{\kappa}, t_{n}(-\kappa) \leqq s_{n} \leqq t_{n}(\kappa)$, so that

$$
\frac{t_{n}(-\kappa)}{2 B_{0} F(n)} \leqq \frac{s_{n}}{2 B_{0} F(n)} \leqq \frac{t_{n}(\kappa)}{2 B_{0} F(n)} .
$$

Let $n \rightarrow \infty$. By (31), the extreme members of the last written inequality tend to 1 , for almost all $x$, and hence

$$
\lim _{n \rightarrow \infty} \frac{s_{n}}{2 B_{0} F(n)}=1 \quad \text { on a set of measure }>1-\delta .
$$

Since $\delta$ is arbitrary,

$$
\text { No }\left\{k, r \mid\left\langle q_{k} x\right\rangle<\frac{f\left(B_{0}^{-1} \log r q_{k}\right)}{r^{2} q_{k}}, 1 \leqq r q_{k} \leqq q_{n}\right\} \sim 2 B_{0} F(n)
$$

for almost all $x$.

We now put $f_{1}(x)=f\left(B_{0}^{-1} x\right)$. Clearly, $f_{1}$ satisfies conditions (12) (or (8)) if and only if $f$ does. We have 


$$
\begin{aligned}
B_{0} F(n) & =B_{0} \sum_{k=1}^{n} f(k)=B_{0} \int_{1}^{n} f(t) d t+O(1)=\int_{1}^{B_{0} n} f_{1}(u) d u+O(1) \\
& =\sum_{k=1}^{B_{0} n} f_{1}(k)+O(1) .
\end{aligned}
$$

Hence by (32),

$$
\text { No }\left\{k, r \mid\left\langle q_{k} x\right\rangle<\frac{f_{1}\left(\log r q_{k}\right)}{r^{2} q_{k}}, 1 \leqq r q_{k} \leqq q_{n}\right\} \sim 2 \sum_{k=1}^{B_{0} n} f_{1}(k)=2 F_{1}\left(B_{0} n\right)
$$

for almost all $x$, and since $f_{1}(t)<1 / 2$ for $t \geqq 0$, we have that

$$
\text { No }\left\{m \leqq q_{n} \mid\langle m x\rangle<\frac{f_{1}(\log m)}{m}\right\} \sim F_{1}\left(B_{0} n\right)
$$

for almost all $x$, the left members of the last two displayed formulas being identical.

Let $z$ be a positive real number, and put

$$
\begin{aligned}
& u(n)=u(n, x)=\text { No }\left\{m \leqq q_{n} \mid\langle m x\rangle<\frac{f_{1}(\log m)}{m}\right\}, \\
& v(z)=v(z, x)=\text { No }\left\{m \leqq e^{B_{0} z} \mid\langle m x\rangle<\frac{f_{1}(\log m)}{m}\right\} .
\end{aligned}
$$

For $x \in G$,

$$
q_{[z+\kappa z \eta]}>e^{B_{0}[z+\kappa z \eta]-\kappa[z+\kappa z]^{\eta}}>e^{B_{0} z} \quad \text { for } z>z_{0}(\kappa),
$$

and

$$
q_{\left[z-\kappa z^{\eta}\right]}<e^{B_{0}\left[z-\kappa z^{\eta}\right]+\kappa\left[z-\kappa z^{\eta}\right]^{\eta}}<e^{B_{0} z} \text { for } z>z_{1}(\kappa) .
$$

Now let $\delta$ be positive, and let $\kappa$ again be so large that $\mathfrak{T} G_{\kappa}>1-\delta$. For $z$ sufficiently large, and for all $x \in G_{k}$,

$$
u\left(\left[z-\kappa z^{\eta}\right]\right) \leqq v(z) \leqq u\left(\left[z+\kappa z^{\eta}\right]\right),
$$

whence

$$
\begin{aligned}
\frac{F_{1}\left(B_{0}\left[z-\kappa z^{\eta}\right]\right)}{F_{1}\left(B_{0} z\right)} \cdot \frac{u\left(\left[z-\kappa z^{\eta}\right]\right)}{2 F_{1}\left(B_{0}\left[z-\kappa z^{\eta}\right]\right)} & \\
& \leqq \frac{v(z)}{2 F_{1}\left(B_{0} z\right)} \leqq \frac{F_{1}\left(B_{0}\left[z+\kappa z^{\eta}\right]\right)}{F_{1}\left(B_{0} z\right)} \cdot \frac{u\left(\left[z+\kappa z^{\eta}\right]\right)}{2 F_{1}\left(B_{0}\left[z+\kappa z^{\eta}\right]\right)} \cdot
\end{aligned}
$$

Now let $z \rightarrow \infty$. By the corollary to Lemma 4 , the first factor in each of the extreme members of this inequality approaches 1 . By (33), the second factor also approaches 1 , except on a set of measure 0 , and hence 


$$
\lim _{z \rightarrow \infty} \frac{v(z)}{2 F_{1}\left(B_{0} z\right)}=1 \quad \text { on a set of measure }>1-\delta .
$$

Since $\delta$ is arbitrary, it follows that for almost all $x$,

$$
\text { No }\left\{m \leqq e^{B_{0} z} \mid\langle m x\rangle<\frac{f_{1}(\log m)}{m}\right\} \sim 2 \sum_{k=1}^{B_{0} z} f_{1}(k) \text {. }
$$

Since

$$
\begin{aligned}
\sum_{k=1}^{B_{0} z} f_{1}(k) & =\int_{1}^{B_{0 z} z} f_{1}(u) d u+O(1)=\int_{1}^{e B_{0} z} f_{1}(\log t) \frac{d t}{t}+O(1) \\
& =\sum_{k=1}^{e B_{0} s} \frac{f_{1}(\log k)}{k}+O(1),
\end{aligned}
$$

this shows that

$$
\text { No }\left\{m \leqq e^{B_{0} z} \mid\langle m x\rangle\left\langle g_{1}(m)\right\} \sim 2 \sum_{k=1}^{e B_{0} z} g_{1}(k) \quad \text { for almost all } x,\right.
$$

where $g_{1}(t)=f_{1}(\log t) / t$. Finally, restricting $z$ to the numbers $B_{0}^{-1} \log n$, with positive integral $n$, we have that

$$
\text { No }\left\{m \leqq n \mid\langle m x\rangle\left\langle g_{1}(m)\right\} \sim 2 \sum_{k=1}^{n} g_{1}(k) \quad \text { for almost all } x .\right.
$$

Since $g_{1}$ can be any function satisfying (11), the theorem is proved.

\section{REFERENCES}

1. K. Knopp, Theory and application of infinite series, London, Blackie and Sons, 1951.

2. W. J. LeVeque, On the frequency of small fractional parts in certain real sequences, Trans. Amer. Math. Soc. vol. 87 (1958), pp. 237-260.

3. P. Lévy, Théorie de l'addition des variables aléatoires, Paris, Gauthier-Villars, 1937.

MATHEMATISChES INSTITUT DER UNIVERSITÄT, Göttingen, Germany 